\title{
The Individual Change in Surface Air Temperature and the Heat Quantity Supplied to the Earth's Surface
}

\author{
By K. Miyakoda \\ Geophysical Institute, Tokyo University \\ (Manuscript received 15 Sept., 195\%)
}

\begin{abstract}
Using the data of surface observation, amounts of temperature change during three hours due to the horizontal advection of air are calculated for every 3 hours during one month of May 1954; and they are compared with observed local changes in temperature.

Further the daily series of the individual changes in temperature are compared with those of the heat supplied to the earth's surface. Then, it is shown that in daytime they are well correlated with each other, and that in later night the correlation is low, though in early night - it is comparatively high. These circumstances are discussed in this paper.
\end{abstract}

\section{Introduction}

It is desired to establish an estimating formula of the vertical flux of sensible heat over the land, which is universally applicable to any case. Concerning this problem several approaches have been made from various points of view. In particular, Brunt (1932) and others attempted to explain the temperature change by the concept of eddy transfer in the lower boundary layer of the atmosphere. Since then, many investigations were performed to determine the eddy conductivity quantitatively, using the data on the time variation of the vertical distribution of temperature specifically measured.

However, the studies which have hitherto been done were mostly confined to the treatment of the data obtained at one observing station. It is apparent that the most part of temperature change is due to the horizontal and the vertical advection of air, and only the remaining part is ascribed to the heating and the cooling of the ground as well as the vertical transfer of heat. In other words, in order to get the real individual change in temperature, we need at least two observing stations. But the measurements adapted for such a purpose have rarely existed. The difficulty in this case is that the time interval used for the calculation of temperature change

Contribution No. 110. can never be taken long, for the air temperature over the land shows the diurnal variation. Therefore the data of routine radiosonde observations are usually not available.

Under these circumstances, making use of the surface observations, we calculated the individual changes in temperature for 3 hours through eliminating the contribution of horizontal advection from the observed local change in temperature. Furthermore, they were compared with the heat quantity supplied to ground surface.

Since this paper is a preliminary report on the investigation of the non-adiabatic change of air, the vertical distribution of individual change in temperature is not included. What is aimed herein is to get a general scope of the vertical transfer of heat, using the comparatively accurate and plentiful data on the surface before studying that of the upper atmosphere.

\section{Estimation of the temperature change due to horizontal advection}

Letting $\Delta T$ and $D T$ be the local and the individual change in temperature, we have the following approximate relation, i.e.,

$$
\Delta T=D T-\int \boldsymbol{V} \cdot \nabla T d t
$$

where the latter part of the right hand side represents the contribution of horizontal ad- 
vection. For a small time interval (in our case, 3 hours), this quantity may approximately be considered as equal to the local change in temperature when the heating or the cooling is absent. Therefore, its magnitude is to be evaluated by subtracting the temperature at starting point from that at the terminal point which is determined by 3 hour advection along the trajectory of an air mass. It is noted that in this case both temperature are for the initial time. Thus, in order to perform this calculation, both the temperature and the wind fields are needed. Furthermore, it is necessary that the observed temperature and wind velocity do never involve the topographic influence, for it induces the complexities in treatment. For meeting these requirements, the Kanto-district in Japan was adopted as the research domain, because this district is mostly occupied by a flat plain with Tokyo located at the centre of it. The temperature change we were concerned with was that of Tokyo. In Fig. 1 , the observing stations around Tokyo are shown, and the range of 3 hour advection.

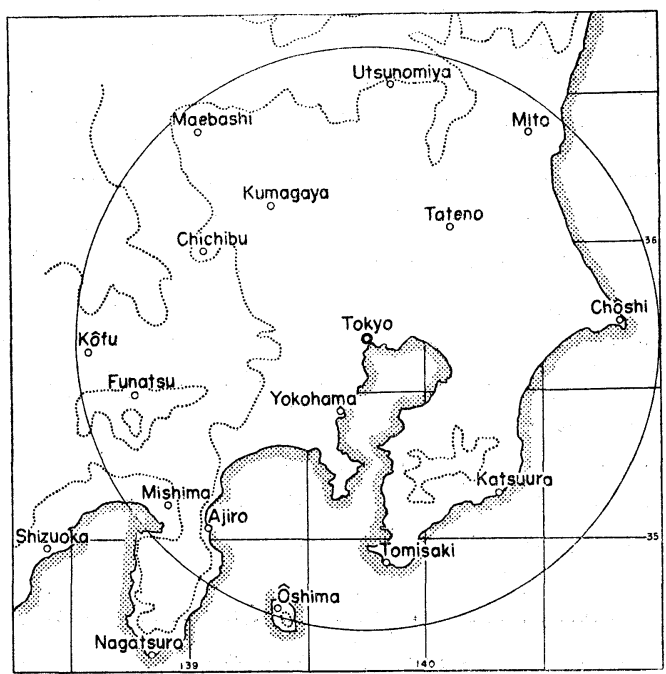

Fig. 1. Geography of Kanto district and the stations used. The circle shows the reach range of an air particle of the speed $10 \mathrm{~m} / \mathrm{sec}$ from Tokyo for 3 hours.

due to the wind of $10 \mathrm{~m} / \mathrm{sec}$ is shown by a circle.

As for the data, one month series of tem- perature and wind velocity observed at every one hour for May 1954 were used. There is no special reason for the adoption of this month. The data of temperature in use were obtained by 3 hour running mean for the elimination of the irregularities of observed values.

The flow pattern at the surface is shown by the short line segments parallel to the wind direction overall the domain. (Fig. 2) Then, a wind trajectory passing through Tokyo was drawn parallel to the segments. (the dashed arrows in Fig. 2) This trajectory is, so to speak, the virtual path which was obtained under the assumption that the wind field was stationary for 3 hours. The actual path was determined by averaging a couple of path vectors thus obtained at the initial and at the terminal time. (the solid arrows in Fig. 2) It was found that this averaging process was inevitable, for the abrupt change of wind direction often occured especially when a local surface front passed over the station concerned. For instance, as in Fig. 2 (a) and (d), the actual paths for $00 \mathrm{~h} \sim 03 \mathrm{~h}$ and $09 \mathrm{~h} \sim 12 \mathrm{~h}$ are utterly different from the virtual ones.

Now the contribution to the local temperature change was estimated by subtracting the the value of temperature at the starting point of the path from that at Tokyo, using only the initial temperature values. The quantity thus obtained for each 3 hours is compared with the observed local change in temperature in Fig. 3, where the black points are for the daytime and the open circles for the nightime. If the observed local changes were completely explained only by this effect, the plotted points in Fig. 3 should be lined up on the straight line crossing the coordinate axis with an angle of $45^{\circ}$. But they, of course, do not in reality. Neverthless this effect is still important.

Before discussing this respect in more detail, it is pointed that $-\int \boldsymbol{V} \cdot \boldsymbol{\nabla} T d t$ appears negative in most cases. This implies that the surface wind blows from a colder to a warmer region, just as suggested by the theory of the land and sea breeze. In other words, the wind in the lower boundary layer of the atmosphere is partly governed by the pressure 


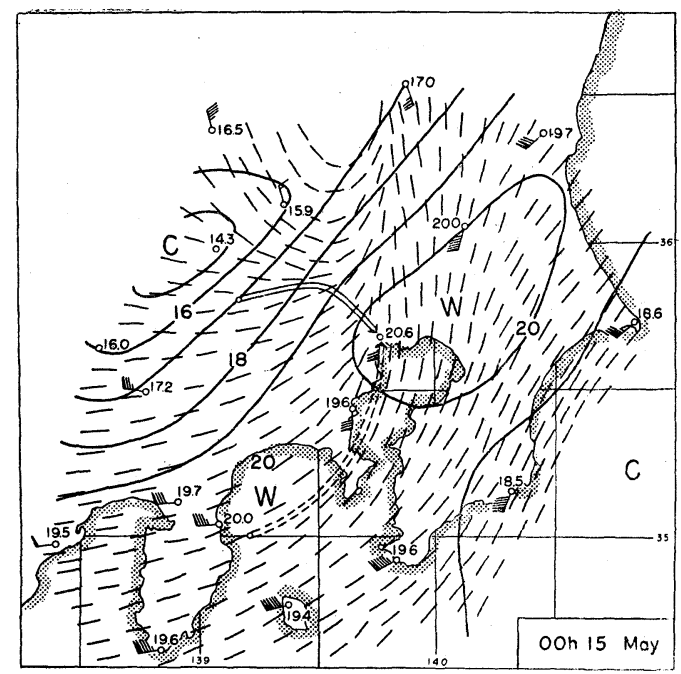

Fig. 2a.

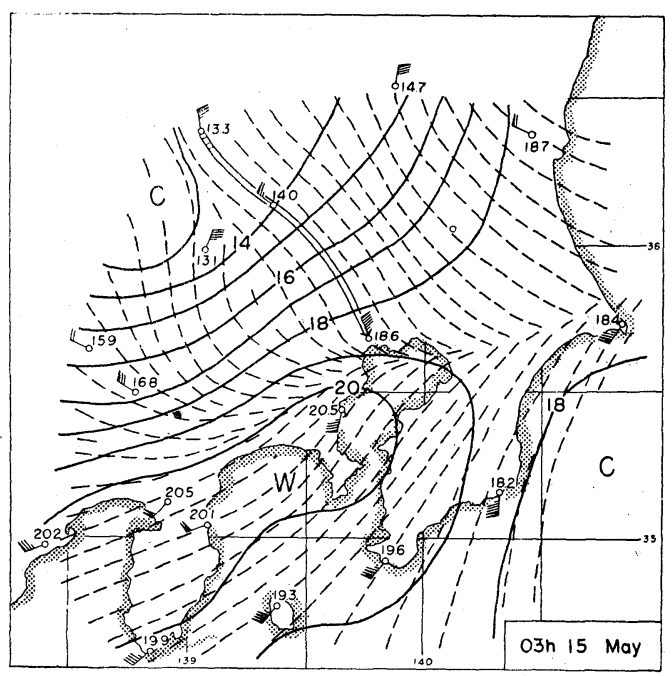

Fig. $2 b$.

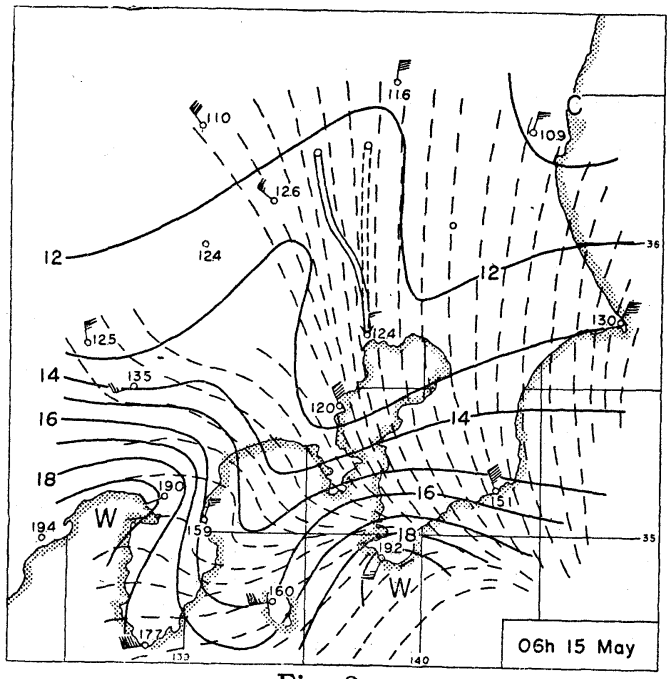

Fig. 2c.

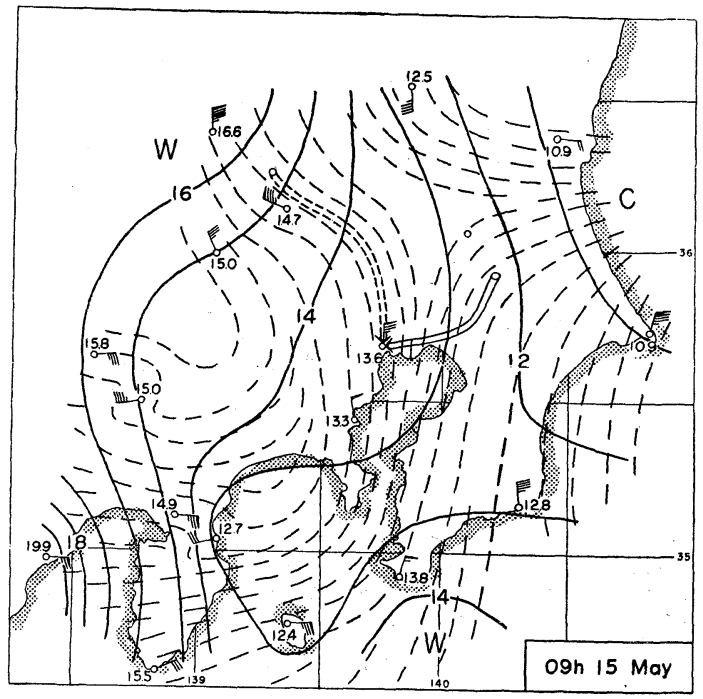

Fig. 2d.

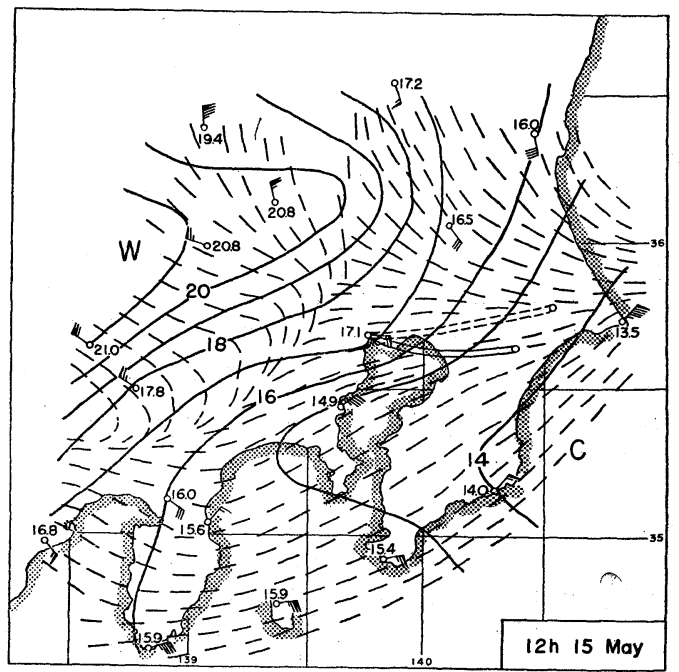

Fig. 2e.

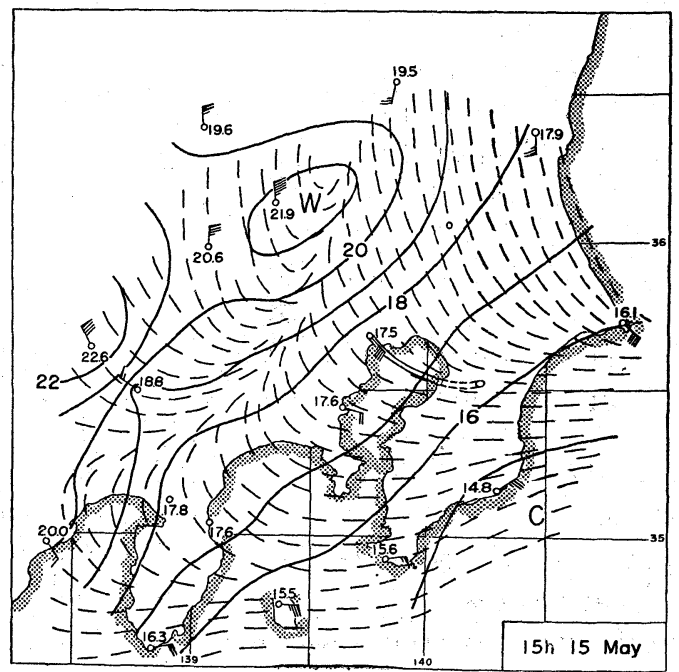

Fig. 2f.

Fig. 2. Examples of the variation of wind (the small line segments) and temperature (the solid contours) fields. The labeled figures are the temperature in ${ }^{\circ} \mathrm{C}$. The solid arrows are the actual trajectory of an air parcel for the preceeding 3 hours, and the dashed arrows the trajectory virtually obtained feathers indicates the wind speed of $5 \mathrm{~m} / \mathrm{sec}$. 


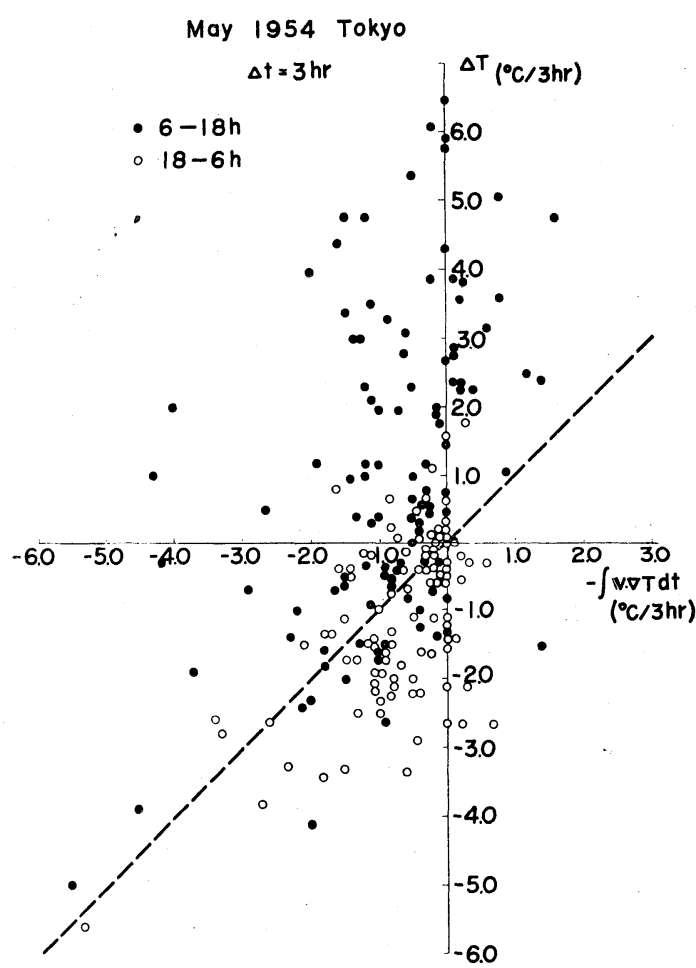

Fig. 3. The observed local change in temperature $\Delta T$ for 3 hour interval at Tokyo against the contribution to temperature change from the horizontal advection of the $\operatorname{air} \int \boldsymbol{V} \cdot \nabla T d t$. The black points are for the daytime and the open circles for the nighttime.

and the Coriolis forces and at the same time it is in close relation with the temperature field. This tendency is also observed in the examples of Fig. 2: even though at one instant the wind seems to blow independently to the temperature field, in the next time it is directed to a warmer region. This may suggest that the free convection due to buoyancy force asserted by Malkus and Stern (1953) and Priestely (1957) is taking place, and that it is important for the vertical transport of heat over the land near the sea.

In Fig. 4, the contribution of horizontal advection is compared with the observed local change of temperature for each day, being classified by the local time. As is seen in this figure, a little before the dawn and the sunset, this contribution occupies the most part of the temperature change. This feature is clearly shown in Fig. 5, where the correlation coefficients between them are presented for

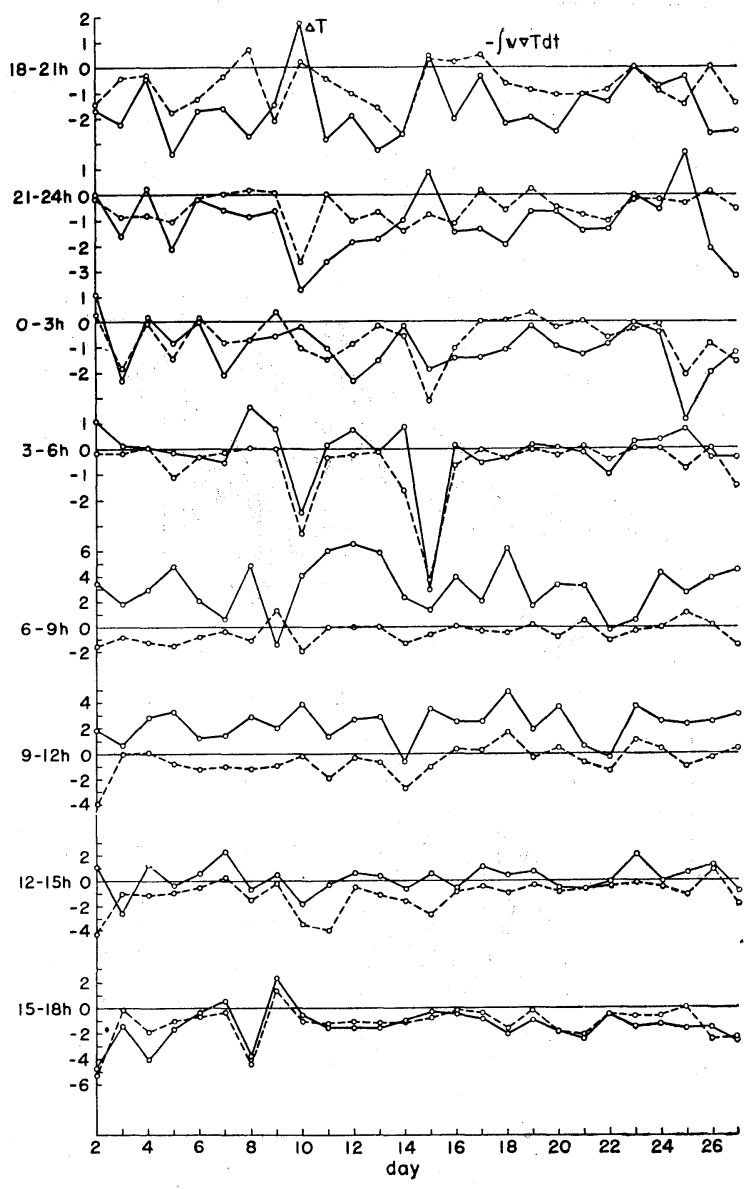

Fig. 4. Daily series of the contribution due to the horizontal advection $-\int \boldsymbol{V} \cdot \boldsymbol{\nabla} T d t$ (dashed line) and the observed local change in temperature $\Delta T$ (solid line), which are classified by the local times.

The scale of ordinate in the daytime is taken twice as that in the nighttime.

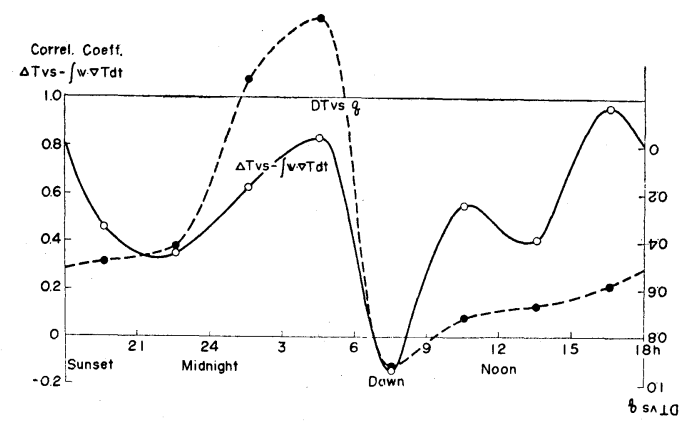

Fig. 5. Diurnal variation of the correlation coefficients between $\Delta T$ and $\int V \cdot \nabla T d t$ (the solid line), and between $D T$ and $q$ (the dashed line), where the scale of the latter coefficient is taken upside down. 
each local time. Another thing which may be known from this figure is that the contributions in the night are comparatively large compared with the total local change and in the daytime they are small.

\section{The heat supplied to the earth's surface}

Our purpose is to compare the individual change of temperature with that of the heat supplied to the ground surface. The determination of the latter quantity was performed in the following way. Letting $Q_{+}$and $Q_{\text {- be the intensities of solar }}$ and terrestrial radiation, respectively, we have the heat quantity $q$ in the following relation;

$$
q=(1-\alpha) Q_{+}-Q_{-},
$$

where $\alpha$ is the albedo of the earth's surface. Practically we used 0.17 as its value according to Fritz' result on the average albedo (1949). As the value of incoming solar radiation $Q_{+}$, the data obtained from Robitzsch pyrheliometer at Tokyo were adopted.

Concerning the outgoing nocturnal radiation $Q_{-}$, the various formulae for its evaluation have been presented. (for instance, Brunt 1932) Among them, we chose the Yamamoto's furmula for clear sky (1950), and for the estimation of the shielding influence of cloud, we adopted the Phillips scheme (1940) as follows,

$$
Q_{-}=\sigma T^{4}(1-c-d \sqrt{e})(1-n \cdot k),
$$

where $\sigma$ is the Stefan-Boltzmann constant, $T$ the absolute temperature, $e$ the water vapour pressure, $n$ the cloudiness and $k$ the index of cloud height. The numerals of the coefficients $c$ and $d$ presented by Yamamoto are 0.51 and $0.066 \mathrm{mb}^{-\frac{1}{2}}$, respectively. The index of cloud height chosen herein are obtained by Phillips as in the following table.

Table 1. The index of cloud height (after Phillips)

\begin{tabular}{c|lll}
\hline Cloud height $(\mathrm{km})$ & 2 & 5 & 8 \\
\hline$k$ & 0.83 & 0.62 & 0.45 \\
\hline
\end{tabular}

Thus we estimated the long wave radiation from the ground $Q_{-}$, using the various data routinely reported in Tokyo. As the result, in accord with what has been commonly conceived, it was found that the dominant effect on the variation of $Q_{\text {- was }}$ the cloud coverage, and the contributions from the variation of temperature and water vapour pressure were rather small. Further it would also be plausible that $Q_{+}$and $Q_{-}$have a sharp negative correlation as in Fig. 6.

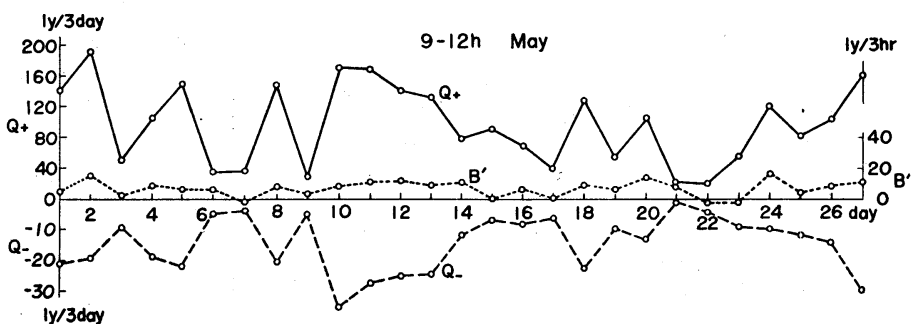

Fig. 6. An example of daily series of the incoming solar radiation $Q_{+}$, the outgoing terrestrial radiation $Q$ - and the heat penesoil $B^{\prime}$, for $9-12 \mathrm{~h}$.

\section{General feature of diurnal variation of various quantities}

In this section, we show the general feature of the diurnal variation of the various quantities related to our problem. In Fig. 7, the monthly mean of the quantities, i.e., the local

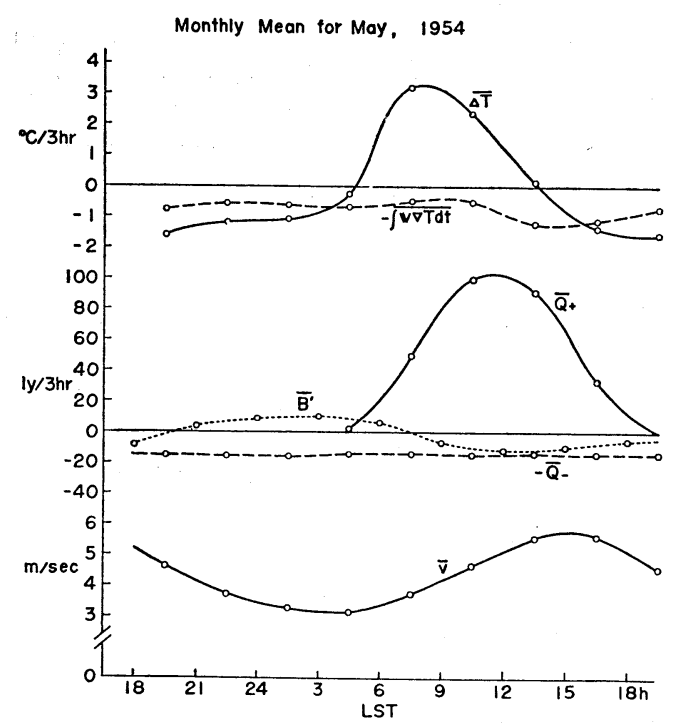

Fig. 7. Diurnal variation of the monthly mean of the various quantities; the local change in temperature $\Delta T$, the contribution due to horizontal advection $\int V \cdot \nabla T d t$, the incoming solar radiation $Q_{+}$, the outgoing terrestrial radiation $Q_{-}$, the heat penetrating into the soil $B^{\prime}$ and the wind speed V. 
change $\Delta T$, the contribution due to horizontal advection $-\int V \cdot \nabla T d t$, the incoming solar radiation $Q_{+}$, the outgoing radiation $Q_{-}$and the wind speed $V$ are shown. It is remarkable that $\Delta T, Q_{+}$and $V$ show the periodical variation while $-\int \boldsymbol{V} \cdot \nabla T d t$ and $Q_{-}$do not.

5. Comparison of daily series of the individual temperature change with the heat

Selection of data

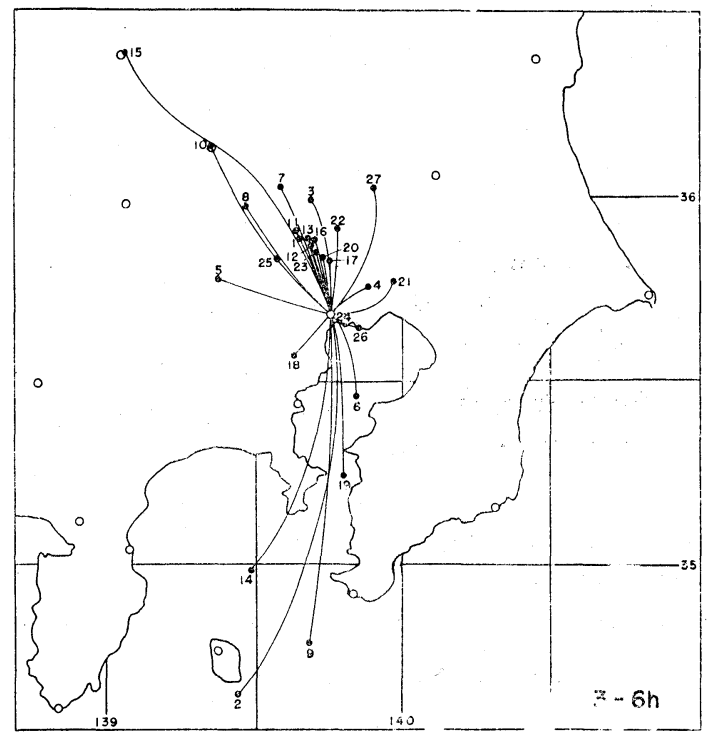

Fig. 8a.

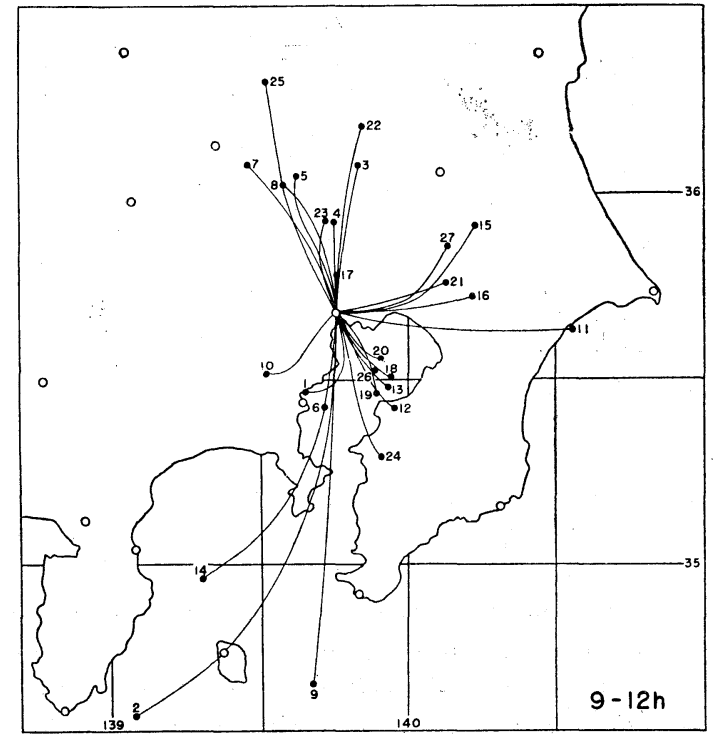

Fig. $8 \mathrm{~b}$.
Before comparing the individual change with the heat quantity, it is necessary to select the suitable data, for our discussion is confined to the modification of air over the land. Namely some air parcels traveled over the land while others come from the sea. In Fig. 8 , the trajectories for 3 hours are shown for the respective local time. As is expected from the theory of land and sea breeze, in the night the air comes mainly from the inland, but in the daytime from the sea.

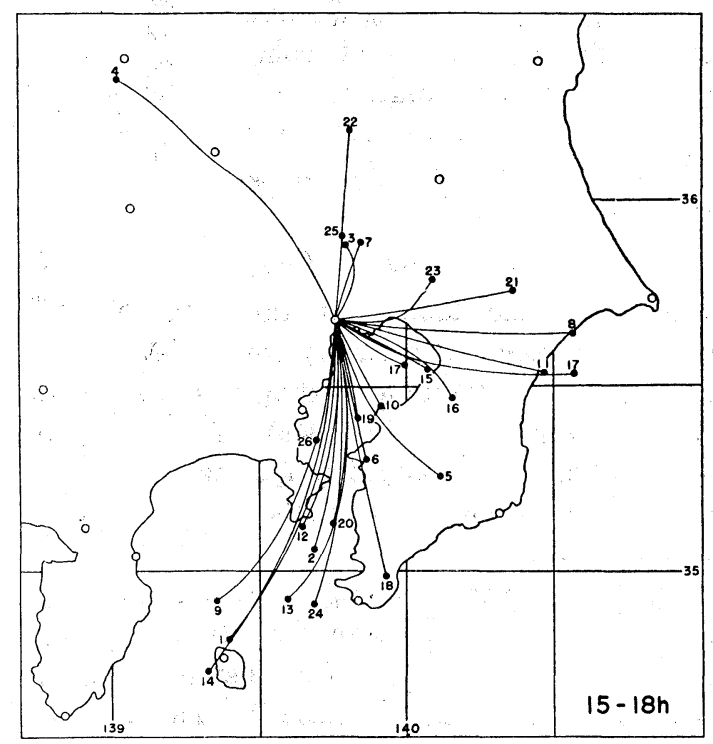

Fig. 8c.

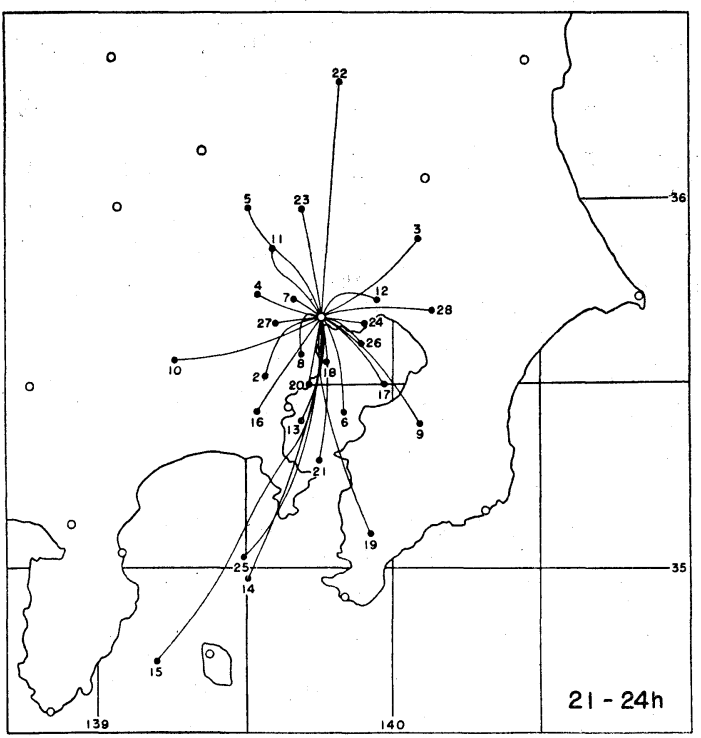

Fig. 8d.

Fig. 8. The path of air particles which reached Tokyo for the respective local time for 3 hours. The figures indicates the dates. 
Comparison

The daily series of the individual change of temperature selected in the above way are
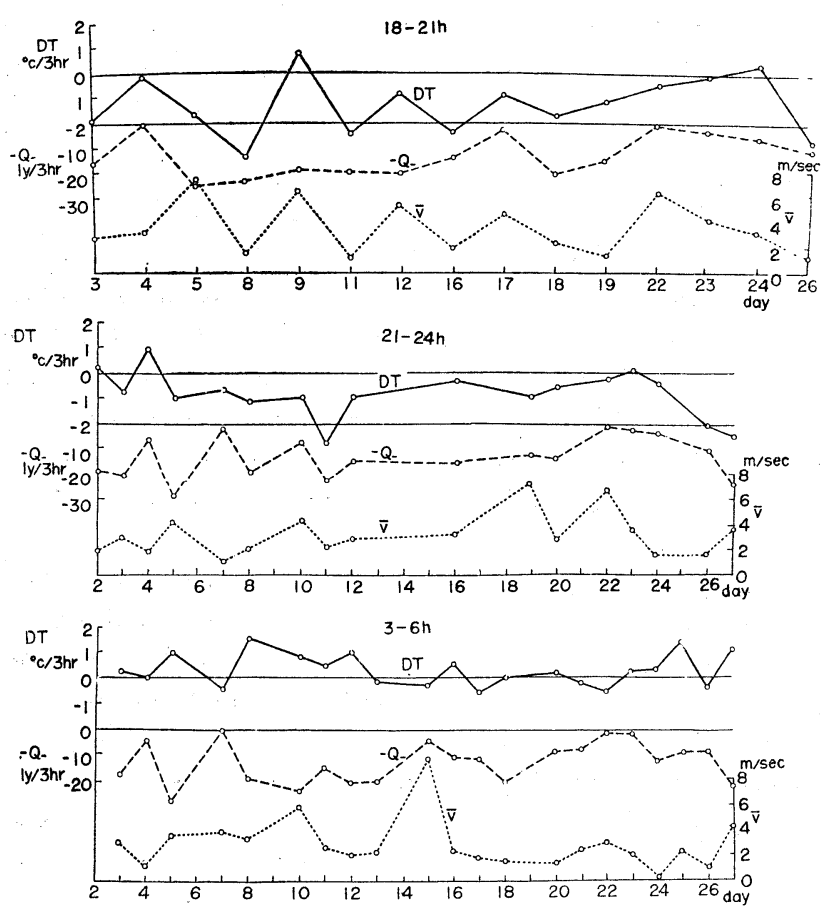

(a) for the nightime $\left(q=-Q_{-}\right)$
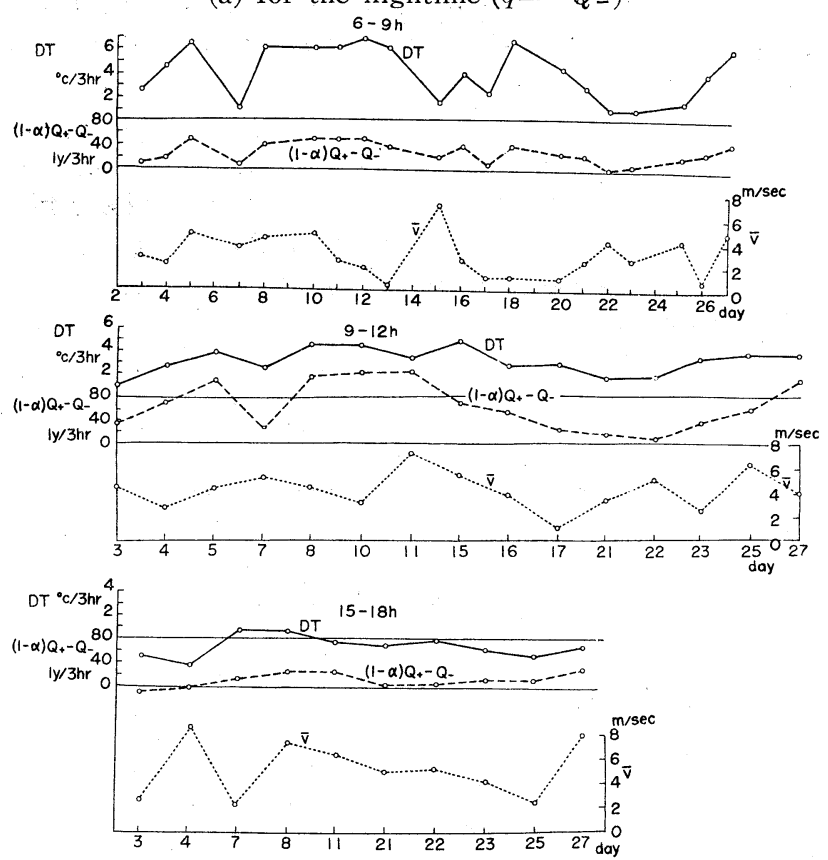

(b) for the daytime $\left(q=(1-\alpha) Q_{+}-Q_{-}\right)$

Fig. 9. Comparison of the individual changes in temperature $D T$ with the heat supplied to the surface $q$ and the wind speed $V$.

(a) for nighttime $\left(q=-Q_{-}\right)$

(b) for daytime $\left(q=(1-\alpha) Q_{+}-Q_{-}\right)$ compared with the heat quantities supplied to the ground surface for each local time in Fig. 9. In the same figure, the wind speeds are also shown for comparison, for the eddy coefficient has been considered in close relation to the wind speed (Petterssen and Swindbank 1947, Takahashi 1948 and Ogura 1949). The data in the left hand side column of Fig. 9 is for the nightime and those in the right for the sunlit hours.

In this figure, the following points are pronounced. (1) For $18-21 \mathrm{~h}, 21$ $-24 \mathrm{~h}$, and for $6-18 \mathrm{~h}, D T$ shows the variation nearly similar to that of $q$. (2) Even in the night when the supplied heat is negative, the temperature sometimes increases. Especially for $18-21 \mathrm{~h}$, the above feature is remarkable when the wind is strong. For $3-6 \mathrm{~h}$, this phenomenon frequently happens, even when the wind blows not so strongly. The counterpart tendency. in the daytime, i.e., the decrease of temperature in the sunlit hours is observed for $15-18 \mathrm{~h}$. (3) The increase of temperature for $6-9 \mathrm{~h}$ is considerably large compared with that for 9-12 $\mathrm{h}$.

\section{Discussion}

The first point mentioned above is very natural. In order to scrutinize this relation, the correlation coefficients between $D T$ and $q$ were calculated and shown in Fig. 5. It is noted that the vertical scale for these coefficients is taken upside-down for the convenience. of comparison. From this figure, it is understood that in the daytime $6-18 \mathrm{~h}$ the correlation coefficients between $D T$ and $q$ are very high, and in the night also they are comparatively high for 18-21 $\mathrm{h}$ and $21-24 \mathrm{~h}$, but in the later night they are low.

Generally speaking, there exist other factors related to temperature change, i.e., the penetrating fraction of heat into the soil and the upward transfer: of heat. The latter element is further: divided into the forced convection due to eddies and the free convection due to bouyancy force. It is generally ac- 
cepted that in the daytime the latter process is predominant and in the nightime the former. From such a point of view together with the results mentioned above, it is likely to say that in the free convection process the heat supplied to the surface is a leading element in temperature change, and in the forced convection process other factors are also important. They would probably be the lapse rate of temperature and the intensity of eddy mixing.

The second point mentioned in the previous section, i.e., the temperature increase in the nightime and the decrease in the sunlit hour, is also related to the above statement. Namely it is conceived that in $18-21 \mathrm{~h}$ when the wind is strong the mixing process would presumably be activated and the intense descending of a warmer air aloft would occur. Strictly to say, as the lapse rate seems to have a close connection with this vertical mixing, it is necessary to know the vertical distribution of temperature for the investigation of this process. In the present research, however, it was not obtained, so the further discussion can not be proceeded. Neverthless, it is almost obvious that in $0-3 \mathrm{~h}$ and $3-6 \mathrm{~h}$ the downward transfer of heat by eddies which is controlled by the stability of the atmosphere seems to play an important role.

The third point is too well known to be explained. This phenomenon is the proof of the existence of free convection especially in the early morning. Concerning the prediction of temperature in the daytime, there has commonly been used the following method. (for example, Ogura 1950, Knizek and Reuter 1950) Assuming that the temperature of air increases keeping its lapse rate as dry adiabatic, the increment of temperature is determined so that the supplied heat quantity may equal to the area on emagram enclosed by the existing vertical profile of temperature and the dry adiabatic line. The physical process involved in this scheme is the free convection. But there may possibly exist another form of heat transfer, i.e., the transfer due to eddy conduction. Thus, the problem is to make clear in what ratio the free convection and the mixing take part in this phenomenon. of the individual change in temperature for 3 hours with that of the heat supplied to the ground surface, which were analysed from the data of surface observation. In order to obtain the individual chang of temperature, the contributions due to horizontal advection of the air were eliminated from the observed local change. As the result, the following points are concluded. The contributions due to horizontal advection of air do not show the systematic diurnal variation, but they individually differ from case to case. A little before the dawn and the sunset they occupy the most part of the local changes in temperature. It is also pronounced that they almost always appear negative. This means that the surface wind blows from a colder to a warmer region.

The individual changes in temperature thus obtained were compared with the heat quantity. The results show that in the daytime they are well correlated with each other, and in the nighttime for $18-21 \mathrm{~h}$ the correlation coefficients are comparatively high. But it was found that in the later nighttime the lapse rate of temperature seems to be important to know the feature of heat transfer.

This is a preliminary research for the estimation of the vertical flux of sensible heat, and the discussions were confined to the surface. It is now being planned to get the similar informations for the upper atmosphere using the radiosonde observation. The difficulty in our problem is that the temperature over the land shows diurnal variation. This implies that the time interval for the calculation of temperature change cannot be taken long. On the other hand, the distance of the radiosonde observing stations are usually $200 \mathrm{~km}$ or more. Therefore, a special device to surmount this difficulty is needed.

\section{Acknowledgements}

The author wishes to express his sincere thanks to Prof. S. Syōno, who gave him many effective suggestions and encouraged him throughout this work. Thanks are due to Dr. Y. Ogura, Messrs. S. Manabe and M. Komabayasi, Tokyo University, who gave him many kind advices and suggestions.

\section{Conclusions and Remarks}

\section{References}

In this paper, we present the comparison

1. Brunt, D. (1932); Notes on radiation in the 
atmosphere. Quart. J. Roy. Meteor. Soc., 58, 389.

2. Brunt, D. (1941): Physical and dynamical meteorology. London Cambridge Univ. Press 368 p.

3. Fritz, S. (1948): The albedo of the ground and atmosphere. B. Amer. Meteor. Soc., 29, 303.

4. Fritz, S. (1951): Solar radiant energy and its modification by the earth and its atmosphere. Compendium Meteor.

5. Knizek, F. und Reuter, H. (1956): Über der Wärmehaushalt der unteren Luftschichten an heiteren Tagen und die Prognose des Maximums der Luft-temperatur. Arch. f. Meteor. Geophys. u. Biokli. s. 324-338.

6. Malkus, J. S. and Stern, M. E. (1953): The flow of a stable atmosphere over a heated island, Part I. J. Meteor. 10, pp. 30- .

7. Ogura, Y. (1949): On the relation between the wind velocity and the diurnal variation of the temperature. Geophys. Notes, Tokyo Univ. 2, No. 11.

8. Ogura, Y. (1950): On the heat transfer in the lower layer of the atmosphere. Geophys. Notes, Tokyo Univ. 3, No. 27.

9. Petterssen, S. and Swinbank, W. C. (1947): Quart. J. Roy. Meteor. Soc., 73.

10. Priestley, C. H. B. (1957): Convection from the earth's surface. Proc. Roy. Soc., Ser. A. 238, no. 1214 Jan. pp. 287-304.

11. Sutton, O. G. (1953): Micrometeorology. New York, McGraw-Hill.

12. Takahashi, K. (1944): The quantitative forecast of the various meteorological elements. (in Japanese) J. Meteor. Soc. Japan, 22.

13. Yamamoto, G. (1952): Effect of radioactive transfer on the vertical distribution of temperature in the atmosphere. Sci. Rep. Tōhoku Univ. Ser. 5 Geophys. 4, 64.

14. Yamamoto, G. (1950). On nocturnal radiation. Sci. Rep. Tōhoku Univ. Ser. 5. Geophys. 2, 27. 